\title{
Interfaces and Extended Structural Defects in Chalcopyrite Thin-Film Solar Cells Studied by Transmission Electron Microscopy
}

\author{
S. S. Schmidt ${ }^{1}$, J. Dietrich ${ }^{1}$, C. T. Koch $^{2}$, B. Schaffer ${ }^{3}$, M. Schaffer ${ }^{3}$, M. Klingsporn ${ }^{4}$, S. Merdes ${ }^{1}$, D. \\ Abou-Ras ${ }^{1}$ \\ 1. Helmholtz-Zentrum Berlin für Materialien und Energie, Berlin, Germany. \\ 2. Ulm University, Institute for Experimental Physics, Ulm, Germany. \\ 3. SuperSTEM, STFC Daresbury Laboratories, Warrington, United Kingdom. \\ 4. Leibniz-Institut für innovative Mikroelektronik, Technology, Frankfurt Oder, Germany.
}

Electronic defects at interfaces between different materials or at extended structural defects such as grain boundaries (GB) or dislocations can deteriorate the performance of a semiconductor device considerably. $\mathrm{Cu}(\mathrm{In}, \mathrm{Ga}) \mathrm{Se}_{2}$ (CIGS) thin-film solar cells consist of several interfaces between individual layers of different materials. In addition, the main light-absorbing layer, the CIGS absorber, exhibits a high density of GBs (i.e., the average grain size is typically smaller than the layer thickness) as well as of dislocations (up to $10^{10} \mathrm{~cm}^{-2}$ in high-efficiency solar cells [1]). Still, CIGS solar cells exhibit highest power-conversion efficiencies of up to $20.8 \%$ [2]. Today, the roles of interfaces and extended structural defects in the solar-cell device are not yet fully understood, although extensive research efforts have been made. We applied various methods in transmission electron microscopy (TEM), such as inline electron holography, electron energy-loss spectroscopy (EELS), as well as energy-dispersive X-ray spectroscopy (EDS) in order to obtain more information about these features.

The interface between the CIGS absorber and the buffer layer, composed of $n$-type $\mathrm{CdS}, \operatorname{In}_{2} \mathrm{~S}_{3}$, or $\mathrm{Zn}(\mathrm{O}, \mathrm{S})$ with thicknesses of few tens of $\mathrm{nm}$, is of special interest since this interface is critical for the formation of the $p-n$ junction. We applied high-resolution EDS to investigate the properties of an atomic layer deposited $\mathrm{Zn}(\mathrm{O}, \mathrm{S})$ buffer layer in a $\mathrm{Cu}(\mathrm{In}, \mathrm{Ga})(\mathrm{Se}, \mathrm{S})_{2}$ solar cell with a high power-conversion efficiency exceeding $16 \%$. We found that the buffer side of the interface with the absorber is enriched with sulfur in a region of a few nm.

In the case of GBs, we generally find a reduced electrostatic potential, i.e., a potential well at the GB core by use of electron holography, see Fig. 1 [3]. The depth of up to few $\mathrm{V}$ and the lateral extension of only 1-2 nm cannot be explained by the presence of excess charges at the GB. However, we were able to estimate a maximum band bending due to excess charge of a few hundred $\mathrm{mV}$, depending on the exact composition, by taking into account charge carrier generation by the electron beam [4]. We also found that strain caused by possible excess charge at the GB is unlikely to give a major contribution to the reduced potential [4]. We identified two possible reasons for the occurrence of the potential wells. The first is a change in composition. At the very same GB that exhibited a potential well we detected a change in composition by use of EELS, see Fig. 1 [3]. The lateral extension of this change in composition is similar to the width of the potential well. An estimate of the electrostatic potential based 
on the measured composition showed that the observed composition profile can indeed cause the dimensions of the measured potential well. The second cause is a reduction in the local atomic density at the GB leading to a reduction in the electrostatic potential. For this estimation, a model $\Sigma 9$ GB in $\mathrm{CuGaSe}_{2}$ was grown and studied by means of electron holography. We performed ab initio calculations on a crystal model of this $\Sigma 9$ GB and compared the resulting electrostatic potential across the GB with the measurement. The measured and computed potential distributions across the GB, exhibit similar dimensions, see Fig. 2 [5]. We showed that, in this case, the reduced local density alone can cause the dimensions of the potential well, and no substantial compositional change has to be assumed.

In the case of dislocations, we also detected a reduced electrostatic potential at the cores [6]. The calculated strain field in combination with multislice simulations showed, however, that the strain is unlikely to cause the potential wells alone. As for GBs, the estimated potential distribution due to a strain field or excess charges at the core exhibits very different dimensions compared with the experimentally observed potential wells. As confirmed by atom-probe tomography, a compositional change at the dislocation core is primarily responsible for the measured potential wells.

\section{References:}

[1] J. Dietrich et al, IEEE Journal of Photovoltaics 2 (2012), p. 364.

[2] Press release: http://www.zsw-bw.de/uploads/media/pi18-2013-ZSW-WorldrecordCIGS.pdf.

[3] D. Abou-Ras et al, Adv. Energy Mater. 2 (2012), p. 992.

[4] S. S. Schmidt, Ph.D. thesis, TU Berlin (2011).

[5] S. S. Schmidt et al, Phys. Rev. Lett. 109 (2012), p. 095506.

[6] J. Dietrich, Ph.D. thesis, TU Berlin (2013).

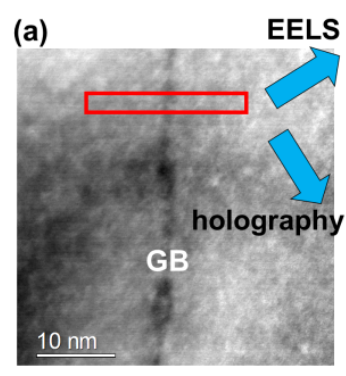

(b)

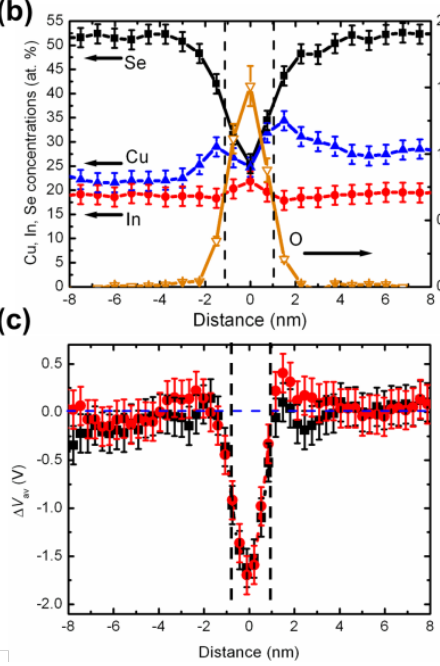

Figure 1. (a) TEM bright field image showing a GB at which the composition (b) and the electrostatic potential (c) were measured by use of EELS and electron holography.

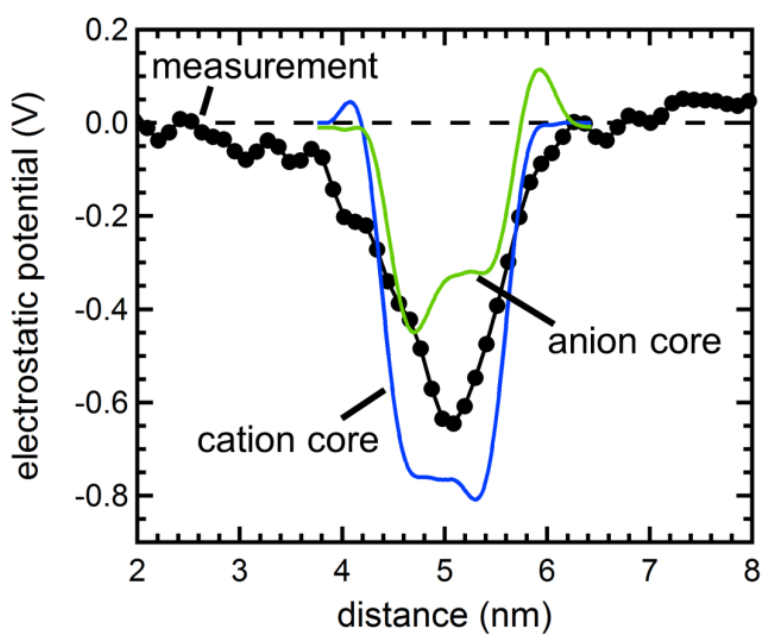

Figure 2. Comparison of the measured electrostatic potential across a $\Sigma 9 \mathrm{~GB}$ in $\mathrm{CuGaSe}_{2}$ with the ones computed for an ab-initio calculated crystal model after application of a band pass filter and assuming a tilt. 